\title{
Philosophiques
}

\section{Guy Bouchard, Le procès de la métaphore. Coll. Brèches, Hurtulbise HMH, 1984, 353 p.}

\section{Marc Turgeon}

Volume 12, numéro 1, printemps 1985

URI : https://id.erudit.org/iderudit/203287ar

DOI : https://doi.org/10.7202/203287ar

Aller au sommaire du numéro

Éditeur(s)

Société de philosophie du Québec

ISSN

0316-2923 (imprimé)

1492-1391 (numérique)

Découvrir la revue

Citer ce compte rendu

Turgeon, M. (1985). Compte rendu de [Guy Bouchard, Le procès de la métaphore. Coll. Brèches, Hurtubise HMH, 1984, 353 p.] Philosophiques, 12(1),

236-241. https://doi.org/10.7202/203287ar d'utilisation que vous pouvez consulter en ligne.

https://apropos.erudit.org/fr/usagers/politique-dutilisation/ 
GUY BOUCHARD, Le procès de la métaphore. Coll. Brèches, Hurtubise HMH, $1984,353 \mathrm{p}$.

$$
\text { par Marc Turgeon }
$$

Deux raisons incitent l'A. à reprendre la problématique de la métaphore. La première est fournie par la persistance de certains problèmes liés à la définition de la métaphore ( " d'où vient le changement d'expression du terme métaphorique ? Comment savoir que le modificateur doit être pris métaphoriquement ? Comment expliquer correctement sa signification ? . . . La métaphore est-elle une énigme ou un phénomène linguistique tout à fait ordinaire ? "(p. 5)). Cette question des limites à assigner à une tentative 
de définition de la métaphore occupe l'A. dans la première partie de son livre. La seconde partie aborde des questions relatives « au rôle de la métaphore dans le discours philosophiques " et au " rôle de la philosophie dans les discours sur la métaphore » (p. 9).

Au plan méthodologique, l'A. part - mais pour tenter de les réconcilier - du débat entre Ricœur et Derrida (La métaphore vive et « La mythologie blanche »). Dans les deux parties de son travail, il examine d'abord les positions respectives de Derrida et de Ricœur avant de proposer une tierce position qui contourne celle - radicale - de Derrida et nuance celle de Ricœur. L'A. indique d'emblée sa sympathie pour un projet de définition restreinte (fondée sur l'usage et le sens commun) de la métaphore. Sans doute y a-t-il plusieurs possibilités de définition de la métaphore (allant de la métaphore comme trope à la métaphore comme synonyme du processus de représentation (p. 5-6)), mais l'A. souhaite que l'on évite l'inflation verbale et, tout en s'attachant à montrer que des conceptions apparemment antagonistes sont souvent complémentaires, se met à la recherche d'une définition de la métaphore, fût-elle normative (p. 12).

Mais avant de tenter une définition de la métaphore il faut contourner l'objection que Derrida fait à la possibilité d'une telle entreprise, liée à « l'impossibilité de parler proprement du non-propre, l'obligation, pour le discours sur la figure, de faire lui-même figure " (p. 15). Le philosophème " métaphore " accueille des métaphores dans sa définition même, ce qui bloque l'accès au propre et rend impossible le contrôle de la polysémie, ce qui est pourtant essentiel à la pensée selon Aristote. La pensée vraie est pensée de la chose même en son sens propre (unique, originel). Le modèle de la métaphore - modèle pour Aristote - , le soleil, est de l'aveu même de celui-ci difficilement connaissable ; son caractère propre n'est jamais assuré, "il n'est jamais présent en propre dans le discours»(p. 19). Ainsi la métaphore, comme son modèle, sera toujours imparfaite et travaillée par des métaphores. À cela, Ricœur opposera que l'opposition que l'on doit retenir n'est pas celle entre sens figuré et sens propre mais celle entre sens figuré et sens littéral : "Nul besoin, dès lors, d'une métaphysique du propre pour justifier la différence entre sens littéral et sens figuré : c'est le discours qui la spécifie » (p. 21). D'autre part, pour parler de la métaphore en philosophie sans verser dans les paradoxes de la déconstruction, il suffit de poser une différence entre les catachrèses, utilisées pour combler une carence de dénomination, " et les métaphores vives créatrices de nouvelles significations » (p. 21). L'examen des présupposés de l'argument de Ricœur convainc l'A. que nous sommes en présence d'une simple différence de point de vue. Derrida tient qu'il en va de la catachrèse comme de la métaphore. Il ne sert donc à rien, comme le fait Ricœur, de lui opposer qu'il faut les distinguer : "Entonner les louanges de la métaphore "vive" n'entame aucunement le fonds de métaphores " mortes » qui constitue, d'après Derrida, les premiers philosophèmes, non plus que cela ne nous assure que la propre théorie de Ricœur ne repose pas, elle aussi, sur des catachrèses » (p. 73). 
Par ailleurs parler de la métaphore suppose que l'on reconnaît « quelque chose " comme métaphore. Comment Derrida échappe-t-il à ce paradoxe ? Selon l'A. il n'y échappe pas. D'une part " sa stratégie manipule certaines conceptions existantes et les utilise comme des hypothèses dont il tire ensuite les conséquences » (p. 74); de ceci semblent résulter certaines incohérences dans le texte derridien, ce qui amène l'A. à souligner que l'accusation de circularité est elle-même circulaire : " en effet, pour montrer que les définitions de la métaphore comportent des métaphores, il faut disposer soimême d'une définition de la métaphore qui permette de reconnaître les métaphores dans les définitions de la métaphore. On notera que si Derrida avait raison, l'impossibilité d'une philosophie de la métaphore ne serait qu'un fragment d'une impossibilité beaucoup plus radicale : celle de toute philosophie du langage. Car il n'est point de lieu non linguistique d'où le philosophe puisse parler du langage » (p. 75).

Il me semble que l'A., compte tenu du parti pris pour la définition de la métaphore qu'il adopte résolument dans son ouvrage aurait pu se dispenser de cette remarque pour s'autoriser à entreprendre sa description des définitions de la métaphore, bien que du point de vue de la confrontation RicœurDerrida il lui faille faire face aux paradoxes que soutient Derrida s'il ne veut pas l'occulter et le mettre en parallèle de sa démarche. Sa réponse à Derrida ne me semble pourtant pas autre chose que cela. Deux remarques, pour justifier cette réserve : 1) on peut difficilement supposer que Derrida cache (ou ignore, ou choisit d'ignorer) une ou des définitions de la métaphore implicitement présentes au fondement de sa déconstruction de la métaphore ; il cherche davantage à "fuguer " sur les définitions proposées, et l'incohérence que lui attribue l'A. (p. 74) ne tient pas à un manque de clarté au niveau des propositions que son texte fonderaient indirectement, mais bien à cette dérive dans la langue des autres ; 2) cette dérive dans la langue des autres est en quelque sorte le propre de la méthode derridienne, s'il est possible de s'exprimer ainsi : il s'agit toujours de traduire et de paraphraser le texte là où il se donne comme démonstration fondée conceptuellement. Ce jeu dans la langue des autres - et avec la sienne - permet à Derrida d'interrompre la synthèse conceptuelle et la hiérarchisation des termes et des discours. C'est pourquoi les paradoxes de la déconstruction marquent en effet l'impossibilité principielle d'une philosophie du langage, non pas cependant au sens où il serait impossible de parler du langage, mais au sens où il serait impossible de fonder un métalangage qui dominerait la langue, de la même manière qu'il cherche à montrer, non qu'il n'y a pas de métaphore, mais que l'on ne peut parler d'elle sans être dedans.

Dans son deuxième chapitre, consacré à la description des théories de la métaphore, l'A. dresse un inventaire des traits pertinents à la définition de celle-ci ; il organise ces traits en un tableau à partir duquel il discute chacune des théories qu'il retient. Cette entreprise lui permet de constater que « les divergences entre les différents auteurs correspondent à la liste des principaux problèmes que doit résoudre une théorie de la métaphore. Ainsi, en ce qui concerne la composition du terme métaphorique, faut-il retenir 
trois facteurs ou deux ? Et, dans ce dernier cas, le facteur que l'on joint au signifiant sera-t-il la signifié ou le désigné ? "(p. 110). Cette grille (p. 8081) permet de décrîre les conceptions existantes de la métaphore, mais aussi " d'en prescrire les conceptions possibles en réalisant l'ensemble des combinaisons permises par les différents traits retenus " (p. 111). Suit un exercice d'évaluation, de comparaison et de réduction des différentes options. Nous savions déjà que "la majorité des auteurs retiennent les trois facteurs" (p. 111) soit le signifiant, le signifié et le désigné. Le raffinement par l'analyse des possibilités de combinaison de ces trois facteurs permet à l'A. de soutenir sept thèses : 1) la métaphore est une expression linguistique appartenant à la classe des expressions caractérisées par la notion d'écart ; 2) l'écart qui caractérise la métaphore se situe au niveau sémantique ; 3) la métaphore comporte un aspect syntagmatique et un aspect paradigmatique ; 4) l'aspect syntagmatique met en cause au minimum deux signes ( $\mathrm{A}$ et $\mathrm{B}$ ) composés chacun d'un signifiant et d'un signifié, l'un étant le modificateur (A) et l'autre le modifié (B) ; 5) l'aspect paradigmatique met en cause un consigne (lieu commun associé, connotation) composé d'un consignifiant et d'un consignifié et substituable au modificateur ; 6) l'écart métaphorique joue à deux niveaux : d'une part entre modificateur et consigne, d'autre part entre modificateur et terme modifié ; l'écart est donc à la fois paradigmatique et syntagmatique, il introduit une double tension (association figurée et impertinence sémantique) ; 7) le fondement de la métaphore est une ressemblance qui, conjointe à une inclusion ou à une exclusion, réduit l'écart en faisant voir, par l'entremise du consigne, sous quels aspects le terme modifié est comme le terme modificateur (p. 119-120). Réduite à sa plus simple expression, la définition de la métaphore irait ainsi : « le nombre minimal de termes pour constituer une métaphore s'élève à trois : modificateur, terme modifié et consigne » (p. 147), « la métaphore est un acte linguistique concret, un événement permettant à une signification nouvelle de fulgurer provisoirement » (p. 169).

Nous sommes remis ici sur la piste du rôle de la métaphore et de son statut en philosophie, discussion qui occupe la deuxième partie du livre. L'A. reprend sa discussion avec Derrida sur les difficultés de fait et de principe liées à l'élaboration d'une métaphorologie. On pourrait, sur le modèle de la métapoétique de Bachelard tenter de montrer comment les images se groupent, s'appellent et se coordonnent. Si, par exemple, on reconstituait la grammaire des métaphores cartésiennes, on reviendrait toujours à celle de la lumière, tout aussi elliptique que celle du soleil chez Aristote. Dans cette perspective : « il n'y aurait plus de vraie métaphore mais à travers une métaphore vraie, la lisibilité assurée du propre » (p. 184). Deux trajets, aussi mortels l'un que l'autre s'offrent à nous : 1) on recherche la métaphore morte, celle qui n'est pas active, dans l'optique d'une " anamnèse intériorisante, relève de la métaphoricité vivante dans une propriété vivante »(p. 184). À l'autre extrême se trouve un désir auto-destructeur qui, en déployant sans limite un philosophème, tente « de lui arracher ses bordures de propriété, 
de faire sauter l'opposition rassurante du propre et du métaphorique . . . » (p. 185).

En plaçant Derrida au plan d'une théorie de la métaphore-mot et en se situant lui-même dans une perspective où la métaphore est une redescription heuristique de la réalité reposant sur la phrase, Ricœur soutient que son option pour la vivacité de la métaphore échappe aux impasses que démontre Derrida. Si la théorie de la métaphore-substitution (mot) " s'accommode de la "relève " du sensible dans l'intelligible, la théorie tensionnelle de la métaphore retire à celle-ci tout privilège, car elle est compatible avec toutes les erreurs calculées susceptibles de faire sens. Ce n'est pas la métaphore qui porte la métaphysique platonisante, mais celle-ci qui s'empare du procès métaphorique à son propre profit " (p. 192) En conséquence, la " métaphore comme fait de langage peut être redécrite par diverses «fictions heuristiques " suscitant de nouvelles métaphores vives ou des métaphores usées puis rénovées, ce qui permet de greffer une nouvelle production conceptuelle sur la production métaphorique » (p. 191).

Ricœur se risque également « à parler de vérité métaphorique pour désigner l'intention " réaliste " qui s'attache au pouvoir de redescription du langage poétique " (cité p. 204) en étendant la théorie de la tension (qui porte sur le sens de l'énoncé) « au rapport référentiel de l'énoncé métaphorique au réel » (p. 204). Ceci permettrait d' « édifier sur la différence reconnue entre modalités de discours une théorie générale des intersections entre sphères du discours, et (de) proposer une interprétation de l'ontologie implicite aux postulants de la référence métaphorique qui satisfasse à cette dialectique des modalités de discours " (cité, p. 210).

L'A. se demande si les positions de Ricœur et de Derrida ne seraient pas cumulatives plutôt qu'exclusives. Il suffirait de relativiser l'objection radicale de Derrida pour rendre cela possible : "C'est l'homme qui donne à son verbe un tour ordinaire ou inusité. Ces deux termes étant relatifs, la recherche de quelque position " en deça de " ou " antérieure "à l'opposition entre l'usité et l'inusité est utopique. Le langage (de la métaphysique) n'est pas tout entier métaphorique au regard de l'être, c'est l'être qui constitue un dépôt tropique » (p. 222). Mais c'est donner raison à Ricœur en endossant sa thèse à l'effet que " les métaphores définissantes que débusque Derrida sont en fait des catachrèses " (p. 222). Derrida ne contesterait sans doute pas que "l'emploi des mots dans le discours" (p. 223) peut produire un effet de sens littéral dans une perspective synchronique, mais sa discussion de la métaphore (comme celle de contexte ou de genre dans d'autres textes) pose l'impossibilité en principe et en fait d'une littéralité, d'une contextualité ou d'une catégorisation univoques, et non pas celle d'un effet, lié à l'usage, de littéralité, de contextualité ou de découpage.

Pour ce qui est de la position de Ricœur sur le postulat de la référence, l'A. utilise les remarques de Searle (p. 224 et s.) sur la différence entre l'existence réelle et l'existence fictive (d'êtres ou de choses auxquels on fait référence) pour souligner son ambiguïté (l'être fictif — ou métaphorique - 
existant sans exister) : " en posant un tel axiome et en y associant la référence, on pourra parler de référence et d'existence à propos de la fiction, mais ce ne sera pas dans le même sens que lorsque ces termes ont trait à la "réalité ", et on ne sera pas pour autant, dans la fiction, "relié aux choses hors de la langue » (p. 226). Mais cette objection n'est pas rédhibitoire car pour l'A. un modèle, même inadéquat ou insuffisant " ou non encore appliqué, aura permis à la pensée de se déployer pendant un certain temps dans son propre élément » (p. 249-250). Un projet intéressant, dans cette perspective, consisterait à discuter la métaphore du point de vue des six fonctions du langage de Jakobson (et une septième, la fonction de support de la pensée de Martinet). Elle permet d'exprimer des sentiments (f. émotive) ; l'expression des pensées et l'invention lexicale (f. désignative) ; elle enrichit l'expression (f. esthétique) ; elle permet d'émouvoir (f. conative) ; elle permet d'élaborer des modèles de connaissance (f. métalinguistique). Mais en voulant, philosophe, montrer que la métaphore est « un principe omniprésent du langage » (p. 251), l'A. ne risque-t-il pas de dissoudre la métaphore en l'incluant dans des processus plus généraux, mettant ainsi en danger son travail de la première partie, consacré à distinguer la métaphore avec un maximum de précision?

Il est à regretter que le désir de l'A. d'ancrer son travail dans l'échange Derrida-Ricœur ait un peu brouillé les objectifs doubles de son livre. La discussion formelle de la métaphore (sans vouloir rien enlever à la richesse et à l'intérêt de celle-ci) semble en effet demeurer, même au terme de ce double itinéraire, à côté de la discussion philosophique du statut cognitif de la métaphore.

Département d'Études littéraires

Université du Québec à Montréal 